\title{
Multi-UAVs Formation Autonomous Control Method Based on RQPSO-FSM-DMPC
}

\author{
Shao-lei Zhou, Yu-hang Kang, Hong-de Dai, and Zhou Chao \\ Department of Control Engineering, Naval Aeronautical and Astronautical University, Yantai 264001, China \\ Correspondence should be addressed to Yu-hang Kang; 492292699@qq.com
}

Received 29 May 2016; Revised 5 August 2016; Accepted 14 August 2016

Academic Editor: Qingsong Xu

Copyright (C) 2016 Shao-lei Zhou et al. This is an open access article distributed under the Creative Commons Attribution License, which permits unrestricted use, distribution, and reproduction in any medium, provided the original work is properly cited.

\begin{abstract}
For various threats in the enemy defense area, in order to achieve covert penetration and implement effective combat against enemy, the unmanned aerial vehicles formation needs to be reconfigured in the process of penetration; the mutual collision avoidance problems and communication constraint problems among the formation also need to be considered. By establishing the virtualleader formation model, this paper puts forward distributed model predictive control and finite state machine formation manager. Combined with distributed cooperative strategy establishing the formation reconfiguration cost function, this paper proposes that adopting the revised quantum-behaved particle swarm algorithm solves the cost function, and it is compared with the result which is solved by particle swarm algorithm. Simulation result shows that this algorithm can control multiple UAVs formation autonomous reconfiguration effectively and achieve covert penetration safely.
\end{abstract}

\section{Introduction}

Multiple UAVs formation control has become an important research direction in the field of UAV control in recent years [1-8], whether in military applications or in civil applications, and it has attracted a lot of attention. Compared with executing the task by single UAV, multiple UAVs formation has significant advantages in terms of time consumption, project selection, task completion rate, and so on. However, as the battlefield environment or formation task changes, the formation or loose degree of UAVs formation needs to be changed, namely, formation reconfiguration. Therefore, it is very important to research a kind of fast, safe, effective, and applicable multiple UAVs formation reconfiguration method [9-14].

Multiple UAVs formation reconfiguration has been widely researched in domestic and foreign regions currently. In order to solve the problem of autonomous formation reconfiguration under the threat of known radar and missile, the literature $[15,16]$ proposed a method that achieves the location optimal configuration of different load UAVs by the plan, reaction, and parameters, then reaches the goal which is the inference of the enemies' radar and missile cooperatively, and ensures the overall safety of the formation, but it did not put forward the effective formation reconfiguration control method for the UAV model. The optimal time control problem of the fixed terminal state constraints has been discussed in detail by the literature $[17,18]$. The optimal time control problem of free terminal state constraints has been discussed in [19-21], but all of them do not consider the distance constraint and collision avoidance constraint. The mathematical description of UAV has been described by the literature [22], and it adopts the optimal control and differential countermeasure theory to analyze whether the external aircraft can influence the collision avoidance among UAVs of the formation. The literature [23] takes artificial potential field method to control the UAV formation so that the UAV formation can avoid the collision among them, but the design of potential field is very complicated, it is only suitable for the collision avoidance of the fixed formation, and it is difficult to be applied to the process of formation reconfiguration. The literature [24] adopts DMPC to solve the collision avoidance problem among UAVs in three-dimensional space, but this method can only calculate the path of collision avoidance, and it is not suitable for the formation problem with clear goal state. The literature [25] maps the formation onto large rigid graph; when the formation passes the obstacle zone, the big rigid graph will 
be divided into many small rigid graphs, the formation passes the obstacle zone by subformation, and this method can only analyze this problem from the angle of system stability, but it does not put forward effective control strategy. In order to solve the multiple UAVs search problem, the literature [26] takes the method of distributed model predictive control that transforms the centralized multiple UAVs optimization decision problem on line to each UAV small scale distributed optimization problem and then adopts the algorithm which is based on particle swarm optimization and Nash optimization achieves iterative solution for each subsystem optimization problem. Based on optimal trajectory generator coupled with a modified sliding controller for tracking the trajectory and avoiding collisions, the literature [27] accomplishes a UAV formation reconfiguration control scheme with autonomous collision avoidance system for application in $3 \mathrm{D}$ space. When facing the uncertainties and obstacles, the literature [28] adopts the learning based model predictive control (LBMPC) to solve the formation reconfiguration problem for a group of $N$ cooperative UAVs forming a desired formation. The literature [29] proposes a distributed linear MPC approach to solve the trajectory planning problem for rotary-wing UAVs, and the simulation results show that this method is valid. Under the constraints of terminal status and of control action energy, the literature [30] puts forward a novel algorithm which is pigeon-inspired optimization to solve the problem of multiple unmanned aerial vehicles formation reconfiguration; compared with particle swarm optimization, this algorithm is better.

This paper elaborates taking distributed model predictive control (DMPC) and finite state machine (FSM) to solve the formation reconfiguration control for multiple UAVs formation which are the mathematic model of UAV, formation model, and different threat constraints and then puts forward revising quantum-behaved particle swarm algorithm (RQPSO) to solve the cost function of DMPC problem, and the particle swarm optimization algorithm solution is compared. The simulation result shows that the algorithm can accomplish formation configuration quickly and efficiently and then achieve covert penetration.

The rest of the paper is organized as follows. Section 2 introduces the UAV motion model and formation model. Main environment threats and constraints will be described in Section 3. The concept of distributed model predictive control and the formation reconfiguration control description is provided in Section 4. Then finite state machine formation management unit and the solution of reconfiguration problem which is revised in quantum particle swarm optimization algorithm are, respectively, presented in Sections 5 and 6. The simulation results and experimental explication are shown in Section 7. Finally, the conclusion and future work are reported in Section 8.

\section{UAV Formation Reconfiguration Model}

2.1. UAV Motion Model. Assuming that there are $N_{v}$ UAVs in the UAV formation, then the UAV formation set can be expressed as $V_{c}=\left\{u v_{i} \mid i=1,2, \ldots, N_{v}\right\}$. All the UAVs can keep the flying height constant; therefore, the UAV centroid motion model after discretization can be described as $[31,32]$

$$
\begin{aligned}
& x_{i}(k+1)=x_{i}(k)+v_{i} \cos \chi_{i}(k) \tau, \\
& y_{i}(k+1)=y_{i}(k)+v_{i} \sin \chi_{i}(k) \tau, \\
& v_{i}(k+1)=v_{i}(k)+\frac{\left(v_{i}^{c}(k)-v_{i}(k)\right)}{\alpha_{v}}, \\
& \chi_{i}(k+1)=\chi_{i}(k)+\frac{\left(\chi_{i}^{c}(k)-\chi_{i}(k)\right)}{\alpha_{\chi}} .
\end{aligned}
$$

$x_{i}, y_{i}, v_{i}, \chi_{i}$ are, respectively, the coordinate, velocity, and track azimuth of UAV in the earth coordinate system. $v_{i}^{c}, \chi_{i}^{c}$ are, respectively, speed command and track azimuth command of UAV. $\alpha_{v}, \alpha_{\chi}$ are, respectively, velocity time constant and track roll angle time constant. $\tau$ is sampling period.

Take the state variable of UAV $u v_{i}$ in $k$ time as $\mathbf{x}_{i}(k)=$ $\left[\begin{array}{llll}x_{i}(k) & y_{i}(k) & v_{i}(k) & \chi_{i}(k)\end{array}\right]^{T}$ and the control variable of UAV $u v_{i}$ in $k$ time as $\mathbf{u}_{i}(k)=\left[\begin{array}{lll}v_{i}^{c}(k) & \chi_{i}^{c}(k)\end{array}\right]^{T}$; then the motion equation of the UAV $u v_{i}$ can be simplified as (2), and the constraint condition can be expressed as (3).

$$
\begin{aligned}
& \mathbf{x}_{i}(k+1)=f_{i}\left(\mathbf{x}_{i}(k), \mathbf{u}_{i}(k)\right), \\
& 0<v_{\min } \leq v_{i} \leq v_{\max }, \\
& \chi_{\min } \leq \chi \leq \chi_{\max }, \\
& \frac{\left(v_{i}(k+1)-v_{i}(k)\right)}{\tau} \leq \Delta v_{\max }, \\
& \frac{\left(\chi_{i}(k+1)-\chi_{i}(k)\right)}{\tau} \leq \Delta \chi_{\max } .
\end{aligned}
$$

Assuming that there is a known reference trajectory so that the UAV formation can follow with it, the reference trajectory satisfies the following [33]:

$$
\begin{aligned}
& x_{r}(k+1)=x_{r}(k)+v_{r}(k) \cos \chi_{r}(k) \tau, \\
& y_{r}(k+1)=y_{r}(k)+v_{r}(k) \sin \chi_{r}(k) \tau, \\
& \chi_{r}(k+1)=\chi_{r}(k)+\omega_{r}(k) \tau .
\end{aligned}
$$

$x_{r}, y_{r}, \chi_{r}$ are, respectively, coordinates and azimuth angle of reference trajectory in the earth coordinate system. $v_{r}, \omega_{r}$ are, respectively, velocity and angular velocity of reference trajectory. Both $v_{r}$ and $\omega_{r}$ are piecewise continuous and uniformly bounded, and they satisfy the following constraint equation:

$$
\begin{aligned}
& 0<v_{r_{\min }}<v_{r}<v_{r_{\max }}, \\
& \omega_{r_{\min }}<\omega_{r}<\omega_{r_{\max }} .
\end{aligned}
$$

2.2. Formation Model. The control strategy of multiple UAVs formation mainly includes the following three: leaderfollower method, virtual-leader method, and behavior control method [34-38]. Because the leader-follower method has 


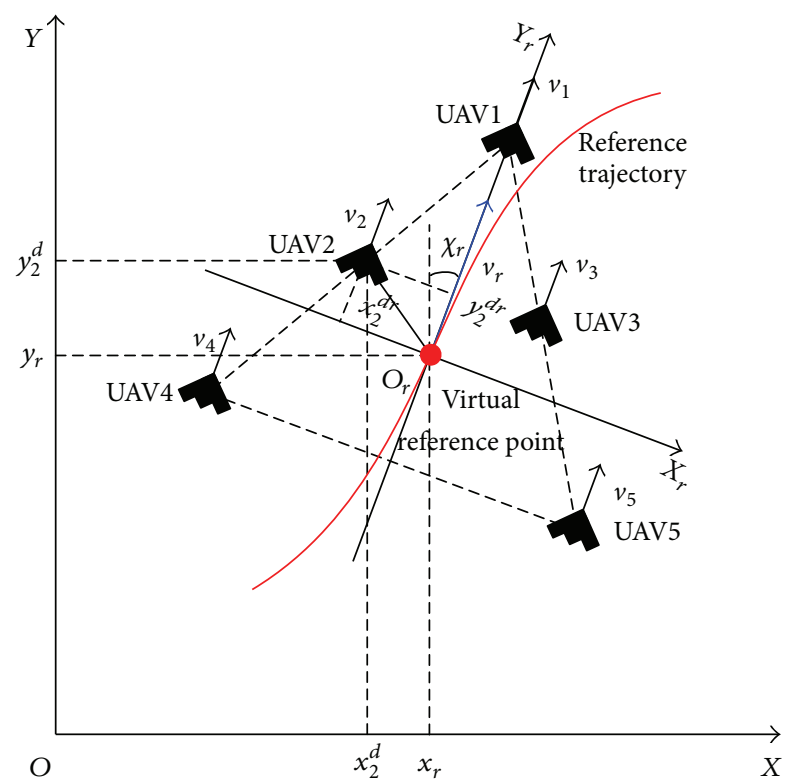

FIGURE 1: Sketch map of formation model.

the error transmission problem, and it is hard to establish the mathematic model for the behavior control method, this paper selects the virtual-leader method. Assuming there exists a reference point which UAVs can follow it, and all UAV s in the formation can achieve the trajectory of reference point in advance or through wireless communication in real-time and keep the relative distance and angle with reference point, there is no reference error among the UAVs. The trajectory reference point coordinate system $X_{r} O_{r} Y_{r}$ is fixedly connected to the reference point $O_{r}$ (Figure 1); thus, we can obtain the desired position of all UAVs in the formation.

$$
\begin{aligned}
{\left[\begin{array}{l}
x_{i}^{d}(k) \\
y_{i}^{d}(k)
\end{array}\right]=} & {\left[\begin{array}{l}
x_{r}(k) \\
y_{r}(k)
\end{array}\right] } \\
& +\left[\begin{array}{cc}
\cos \chi_{r}(k) & \sin \chi_{r}(k) \\
-\sin \chi_{r}(k) & \cos \chi_{r}(k)
\end{array}\right]\left[\begin{array}{c}
x_{i}^{d r}(k) \\
y_{i}^{d r}(k)
\end{array}\right] .
\end{aligned}
$$

$x_{i}^{d r}, y_{i}^{d r}(k)$ are, respectively, the relative distance between the desired position of $u v_{i}$ and trajectory reference point in $k$ time.

\section{Description of Formation Reconfiguration}

3.1. Threat Description. In the process of penetration multiple UAV s formation will face a variety of threats, this paper takes the early warning radar, short-range air defense radar, and no fly zone (including fixed obstacles, fixed threats, and mobile threats), assuming that all the UAVs in formation are able to detect enemy early warning radar and air defense radar, and they carry jamming equipment which is used to interfere in the early warning radar and air defense radar.

3.1.1. Threat of Early Warning Radar. As shown in Figure 2, assuming that the early warning radar's position is $\left(x_{\mathrm{je}}, y_{\mathrm{je}}\right)$,

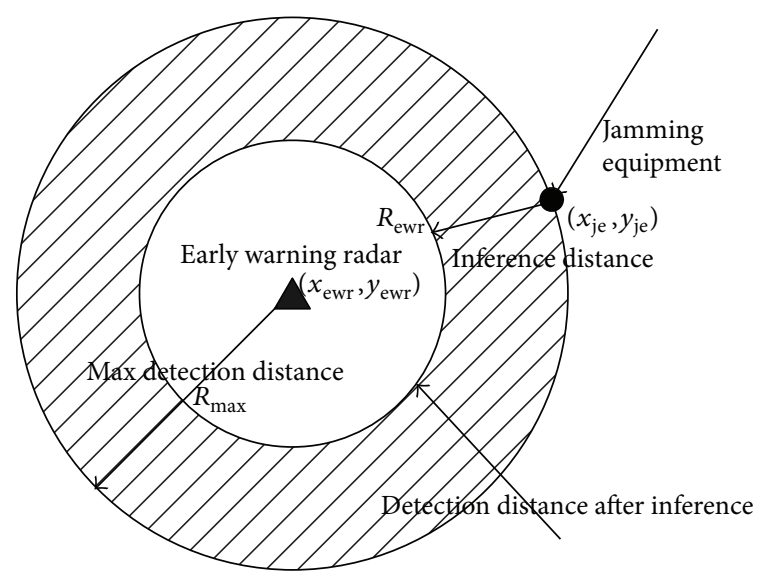

FIGURE 2: Sketch map of early warning radar and interference.

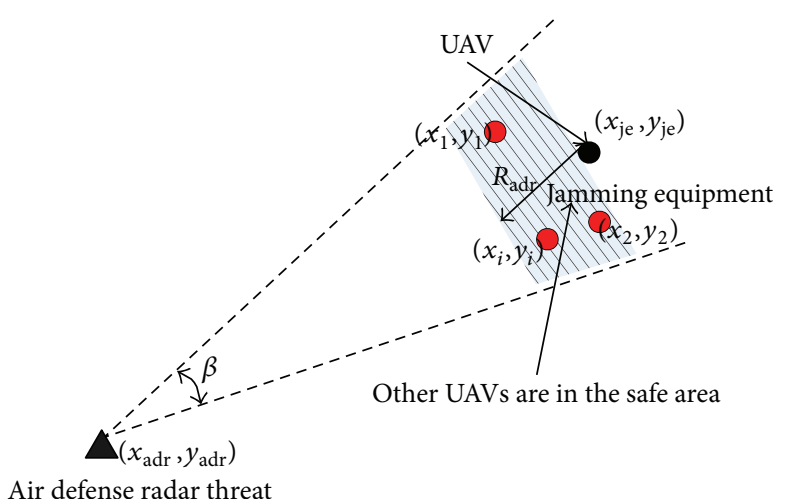

FIGURE 3: Sketch map of air defense radar and interference.

the early warning radar jamming equipment position is $\left(x_{\mathrm{je}}, y_{\mathrm{je}}\right)$, the effective interference distance of early warning radar jamming equipment is $R_{\text {ewr }}$, and the max detection radium of early warning radar is $R_{\max }$, when a certain UAV is interfering the early warning radar, its detection radium can be described as $[39,40]$

$$
\begin{aligned}
R & = \begin{cases}R_{\mathrm{max}}, & \rho_{\mathrm{je}} \geq R_{\mathrm{max}}, \\
\rho_{\mathrm{je}}, & \rho_{\mathrm{je}} \leq R_{\mathrm{max}},\end{cases} \\
\rho_{\mathrm{je}} & =\left(\left(x_{\mathrm{ewr}}-x_{\mathrm{je}}\right)^{2}+\left(y_{\mathrm{ewr}}-y_{\mathrm{je}}\right)^{2}\right)^{1 / 2}-R_{\mathrm{ewr}} .
\end{aligned}
$$

3.1.2. Threat of Air Defense Radar. As shown in Figure 3, assuming that the air defense radar position is $\left(x_{\mathrm{adr}}, y_{\mathrm{adr}}\right)$, the air defense radar jamming equipment position is $\left(x_{\mathrm{je}}, y_{\mathrm{je}}\right)$, the position of $u v_{i}$ is $\left(x_{i}, y_{i}\right)$, the effective interference distance of air defense radar jamming equipment is $R_{\mathrm{adr}}$, the max detection radium and angle of air defense radar are, respectively, 


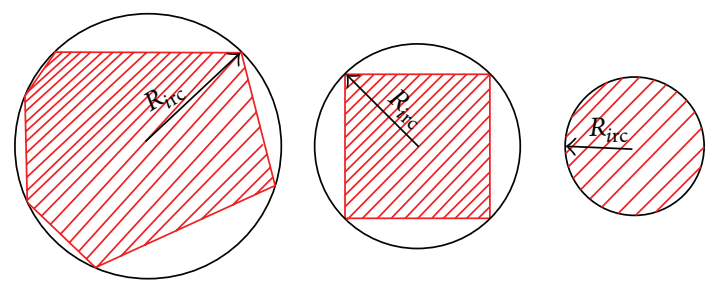

FIGURE 4: Sketch map of nonfly zone.

$R_{\max }$ and $\beta$, then the condition which UAVs can be in the safe zone is $[39,40]$

$$
\begin{aligned}
& d_{s}=\rho_{i a}-\rho_{j a}-R_{\mathrm{adr}}>0 \\
& c s_{s}=\frac{\left(\rho_{i a}^{2}+\rho_{j a}{ }^{2}-\rho_{i j}{ }^{2}\right)}{2 \rho_{i a} \rho_{j a}} \geq \cos \left(\frac{\beta}{2}\right) \\
& \rho_{i a}=\left(\left(x_{\mathrm{adr}}-x_{i}\right)^{2}+\left(y_{\mathrm{adr}}-y_{i}\right)^{2}\right)^{1 / 2}, \\
& \rho_{j a}=\left(\left(x_{\mathrm{adr}}-x_{\mathrm{je}}\right)^{2}+\left(y_{\mathrm{adr}}-y_{\mathrm{je}}\right)^{2}\right)^{1 / 2}, \\
& \rho_{i j}=\left(\left(x_{\mathrm{je}}-x_{i}\right)^{2}+\left(y_{\mathrm{je}}-y_{i}\right)^{2}\right)^{1 / 2} .
\end{aligned}
$$

3.1.3. Nonfly Zone. Once multiple UAVs formation encounters nonfly zones or obstacles, they can only be able to keep away from them, as shown in Figure 4; in order to simplify the problem, the nonfly zones or obstacles are replaced by their minimum circumnavigations in this paper; assuming that the minimum circumnavigations of nonfly zone position and radium are, respectively, $\left(x_{i \mathrm{rc}}, y_{\text {irc }}\right)$ and $R_{\text {irc }}$, then the condition which UAV san be in the safe zone is

$$
d_{i \mathrm{nz}}=\left(\left(x_{i}-x_{i \mathrm{rc}}\right)^{2}+\left(y_{i}-y_{i \mathrm{rc}}\right)^{2}\right)^{1 / 2}-R_{i \mathrm{rc}}>0 .
$$

3.2. Constraint Condition Construction of UAVs Formation. On the one hand, because UAVs formation faces a variety of threats, only to avoid these threats, the UAVs formation can achieve covert penetration, reach the designated zone, and implement effective attack on enemy targets. On the other hand, because UAVs formation needs to avoid collision among the UAVs in the formation and maintain normal communication in real-time in the process of flight, it only keeps a certain distance between one UAV and another UAV, and then the formation can fly safely. Based on the above situation, the constraints condition can be constructed as follows.

3.2.1. Threat Constraint of Early Warning Radar. Assuming that there are $N_{\text {ewr }}$ enemy early warning radars ewr ew $_{j}(j=$ $1,2, \ldots, N_{\text {ewr }}$ ) detected by UAVs in the formation in $k$ time, their position can be expressed as $\left(x_{\mathrm{ewr}_{j}}, y_{\mathrm{ewr}_{j}}\right)$, and the detection radium after interference is $R_{j}$, according to Figure 2, the UAV in the formation can ensure that it is not found by the early warning radar only if it is beyond the early warning radar detection distance after interference; thus, we can construct the threat constraint condition when UAV $i$ faces the early warning radar $j$ as follows:

$$
\begin{aligned}
& h_{i}^{\mathrm{ewr}}\left(x_{i}(k), u_{i}(k)\right) \\
& \quad=R_{j}+R_{\mathrm{ewr}}-\left(\left(x_{i}-x_{\mathrm{ewr}_{j}}\right)^{2}+\left(y_{i}-y_{\mathrm{ewr}_{j}}\right)^{2}\right)^{1 / 2} \\
& \quad>0 .
\end{aligned}
$$

3.2.2. Threat Constraint of Air Defense Radar. Assuming that there are $N_{\mathrm{adr}}$ enemy air defense $\operatorname{radars} \operatorname{adr}_{j}(j=$ $1,2, \ldots, N_{\text {adr }}$ ) detected by UAVs in the formation inktime, their position can be expressed as $\left(x_{\mathrm{adr}_{j}}, y_{\mathrm{adr}_{j}}\right)$, and the safe distance and angle cosine after interference are, respectively, $d_{s_{j}}$ and $c s_{s_{j}}$, according to Figure 3, the UAV in the formation can ensure that it is not found by air defense radar only if it is in the safe zone; thus, we can construct the threat constraint condition when UAV $i$ faces the air defense radar $j$ as follows:

$$
\begin{aligned}
& h_{i}^{\mathrm{adr} 1}\left(x_{i}(k), u_{i}(k)\right) \\
& \quad=d_{s_{j}}+R_{\mathrm{adr}}-\left(\left(x_{i}-x_{\mathrm{adr}_{j}}\right)^{2}+\left(y_{i}-y_{\mathrm{adr}_{j}}\right)^{2}\right)^{1 / 2} \\
& \quad>0, \\
& h_{i}^{\mathrm{adr} 2}\left(x_{i}(k), u_{i}(k)\right)=c s_{s_{j}}(k)-\cos \frac{\beta}{2} \leq 0 .
\end{aligned}
$$

3.2.3. Threat Constraint of Nonfly Zone. Assuming that there are $N_{\text {nfz }}$ nonfly zone $\mathrm{nfz}_{j}\left(j=1,2, \ldots, N_{\mathrm{nfz}}\right)$ detected by UAVs in the formation in $k$ time, and the minimal circumnavigation of nonfly zone can be expressed as $\left(x_{i \mathrm{rc}_{j}}, y_{i \mathrm{rc}_{j}}\right)$, according to Figure 4 , the UAVs in the formation can ensure that they are safe only if they do not fly across the nonfly zones; thus, we can construct the threat constraint condition when UAV $i$ faces the nonfly zone $j$ as follows:

$$
\begin{aligned}
& h_{i}^{i \mathrm{rc} 1}\left(x_{i}(k), u_{i}(k)\right) \\
& \quad=d_{i \mathrm{nz}_{j}}-\left(\left(x_{i}-x_{i \mathrm{rc}_{j}}\right)^{2}+\left(y_{i}-y_{i \mathrm{rc}_{j}}\right)^{2}\right)^{1 / 2}>0 .
\end{aligned}
$$

3.2.4. Collision Free Constraint. In order to ensure that UAVs in the formation avoid collision, the distance between every two UAVs in the formation needs to be greater than the safety distance $D_{\text {safe }}$; thus, we can construct the distance constraint condition as follows:

$$
\begin{aligned}
& d^{i, j}(k) \geq D_{\text {safe }} \quad\left(\forall_{i \neq j}, i, j \in\left\{1, \ldots, N_{v}\right\}\right) \\
& d^{i, j}(k)=\left(\left(x_{i}(k)-x_{j}(k)\right)^{2}+\left(y_{i}(k)-y_{j}(k)\right)^{2}\right)^{1 / 2} .
\end{aligned}
$$

3.2.5. Communication Distance Constraint. Multiple UAVs formation needs to receive many commands or information during missions, like the command from the upper, change task, all UAVs' state information, and so on, so they must keep normal communication in real-time, and owing to the 


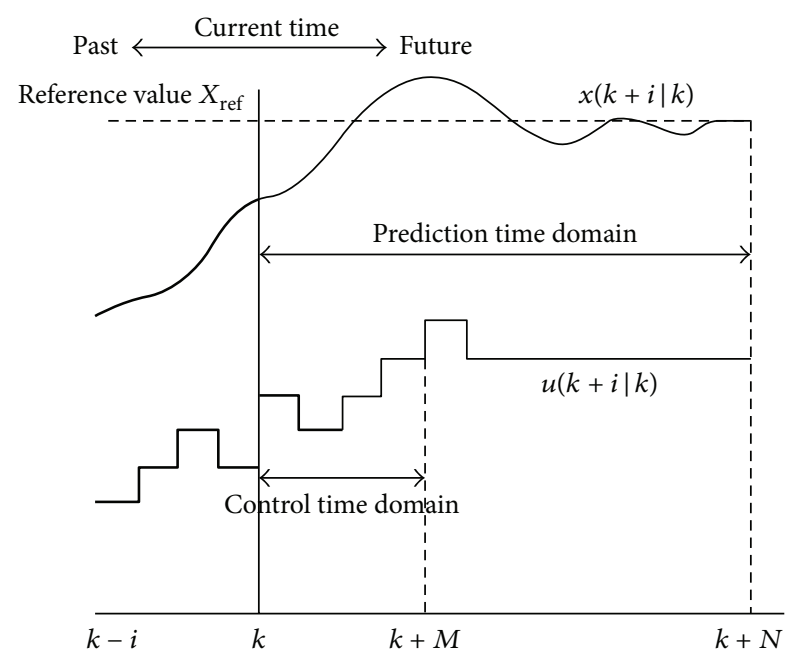

FIGURE 5: Schematic diagram of model predictive control.

communication distance of device being limited, so it is necessary to ensure that the distance between two UAVs is less than the communication security distance $D_{\text {com }}$; thus, we can construct the distance constraint condition as follows:

$$
d^{i, j}(k) \leq D_{\text {com }} \quad\left(\forall_{i \neq j}, i, j \in\left\{1, \ldots, N_{v}\right\}\right) .
$$

\section{Distributed Model Predictive Control (DMPC) Strategy}

4.1. Model Predictive Control (MPC). Model predictive control is also called receding horizon control; its basic principles can be summed up in three aspects: prediction model, rolling optimization, and feedback correction. Since MPC is a finite time domain optimization, not a global optimization strategy, its performance of anti-interference and robustness are very strong [24]. The process of MPC algorithm is shown in Figure 5. follows:

The typical MPC algorithm can be simply described as

(1) Assuming that the system state is $x^{*}(k)$ at $k$ time, thus we can forecast the future output of the system based on the past input.

(2) Select a certain performance index to solve problem online; then the system open loop optimal control input sequence in $N$ prediction of time domain is $\mathbf{u}^{*}(k)=\left[u^{*}(k \mid k), u^{*}(k+1 \mid k), \ldots, u^{*}(k+N-1 \mid k)\right]$.

(3) Select the first item of the system open loop optimal control input sequence $\mathbf{u}^{*}(k)$ as the input of time $k$, namely, $u^{*}(k \mid k)$, and then act in the system.

(4) Repeat the above steps in the new time domain at $k+1$ time.

4.2. Structure of Multiple UAVs Formation Reconfiguration Based on DMPC. The reconfiguration controller needs to optimize all the control inputs in traditional centralized formation reconfiguration control method, the calculation is very large, and it has no scalability [39]. Because these UAVs in the formation are independent, from the view of dynamic characteristics, they are decoupling, so formation reconfiguration can be achieved by the necessary information; thus, the DMPC control architecture can be adopted. Every UAV will be equipped with a MPC controller which can also achieve the information interaction under DMPC control architecture; thus, the overall formation behavior can be described by all subsystems, so the state equation of formation system can be described as [26, 41, 42]

$$
f(\mathbf{x}(k), \mathbf{u}(k))=\left[f_{i}\left(\mathbf{x}_{i}(k), \mathbf{u}_{i}(k)\right) \quad f_{2}\left(\mathbf{x}_{2}(k), \mathbf{u}_{2}(k)\right) \cdots f_{N_{v}}\left(\mathbf{x}_{N_{v}}(k), \mathbf{u}_{N_{v}}(k)\right)\right] .
$$

Further, the overall cost function of formation can be expressed as

$$
\begin{array}{r}
J(\mathbf{X}(k), \mathbf{U}(k))=\sum_{i=1}^{N_{v}} \gamma_{i} J_{i}\left\{\mathbf{X}_{i}(k),\left\{\mathbf{X}_{j \neq i}(k)\right\} \mid \mathbf{U}_{i}(k)\right\} \\
\left(j=1,2, \ldots, N_{v}\right), \\
J(k)=\sum_{s=1}^{N-1}\left\|e_{i}(k+s \mid k)\right\|_{\mathbf{Q}}^{2}+\left\|e_{i}(k+N \mid k)\right\|_{Q^{\prime}}^{2}+\alpha \\
\cdot\left[\sum_{j \neq i}^{N-1} \sum_{s=0}\left\|e_{i}(k+s \mid k)\right\|_{Q^{2}}^{2} \sum_{j \neq i}\left\|e_{i}(k+N \mid k)\right\|_{Q^{\prime}}^{2}\right], \\
\mathbf{X}_{i}(k) \\
=\left\{\mathbf{x}_{i}(k \mid k), \mathbf{x}_{i}(k+1 \mid k), \ldots, \mathbf{x}_{i}(k+N-1 \mid k)\right\},
\end{array}
$$

$$
\begin{aligned}
& \mathbf{U}_{i}(k) \\
& \quad=\left\{\mathbf{u}_{i}(k \mid k), \mathbf{u}_{i}(k+1 \mid k), \ldots, \mathbf{u}_{i}(k+N-1 \mid k)\right\}, \\
& \left\{\mathbf{X}_{j \neq i}(k)\right\}=\left\{\mathbf{X}_{j}(k) \mid j \neq i\right\}, \\
& e_{i}(k+s \mid k)=\mathbf{X}_{i}(k+s \mid k)-\mathbf{X}_{i d}(k+s \mid k), \\
& s=1, \ldots, N .
\end{aligned}
$$

$\mathbf{X}_{i}(k), \mathbf{U}_{i}(k)$ are, respectively, the $N$ step prediction state and $N$ step prediction control input of $i$ th UAV. $\left\{\mathbf{X}_{j \neq i}(k)\right\}$ are $N$ step prediction state set of other UAVs. $\gamma_{i}$ is weight coefficient. $\alpha>0,\left\|e_{i}(k+N \mid k)\right\|_{\mathrm{Q}^{\prime}}^{2}$ and $\sum_{j \neq i}\left\|e_{j}(k+N \mid k)\right\|_{\mathrm{Q}^{\prime}}^{2}$ can reflect the time cost of the overall formation reconfiguration: namely, demanding that every UAV does not deviate from the final desired position too far; thus, the reconfiguration 
control task can be completed as soon as possible. Here the optimization problem can be decomposed into $N_{v}$ small scale local finite time domain optimization problems; thus, the local optimization control model for $i$ th subsystem can be expressed as

$$
\begin{array}{ll}
\mathbf{U}_{i}^{*}(k)=\arg \min _{\mathbf{U}_{i}(k)} & J_{i}\left\{\mathbf{X}_{i}(k),\left\{\mathbf{X}_{j \neq i}(k)\right\} \mid \mathbf{U}_{i}(k)\right\} \\
\text { s.t. } \quad & \mathbf{x}_{i}(k+s+1 \mid k) \\
& =f_{i}\left(\mathbf{x}_{i}(k+s \mid k), \mathbf{u}_{i}(k+s \mid k)\right) \\
( & \left.=0,1, \ldots, N-1 ; i=1,2, \ldots, N_{v}\right) \\
& \mathbf{x}_{i}(k \mid k)=\mathbf{x}_{i}(k) \\
& \mathbf{x}_{i}(k+s \mid k) \in \Xi_{i} \\
& \mathbf{u}_{i}(k+s \mid k) \in \Theta_{i} .
\end{array}
$$

Assuming that all the UAVs can be able to maintain the normal communication under the communication constraint, other UAVs' states $\left\{\mathbf{X}_{j \neq i}(k)\right\}$ can be obtained by communication; thus, the local optimization problem of $i$ th subsystem is relevant to its own state $\mathbf{X}_{i}(k)$, the other UAVs' states $\left\{\mathbf{X}_{j \neq i}(k)\right\}$, and its own control input $\mathbf{U}_{i}(k)$, the scale of optimization problem is greatly reduced, and ith UAV's control input can be obtained by solving this optimization problem. Combined with the threat constraints, the overall reconfiguration optimization model of UAV formation can be expressed as

$$
\begin{aligned}
J(\mathbf{X}, \mathbf{U})=\sum_{i=1, i \neq j}^{i=N_{v}} \min _{i}^{*} \quad & g_{i} J\left(\mathbf{X}_{i},\left\{\mathbf{X}_{j \neq i}(k)\right\}, \mathbf{U}_{i}\right) \\
\text { s.t. } \quad & \mathbf{x}_{i}(k+s+1 \mid k) \\
& =f\left(\mathbf{x}_{i}(k+s \mid k), \mathbf{u}_{i}(k+s \mid k)\right) ; \\
& \mathbf{x}_{i}(k \mid k)=\mathbf{x}_{i}(k) ; \\
& \mathbf{u}_{i}(k \mid k)=\mathbf{u}_{i}(k) ; \\
& v_{i}(k+s \mid k) \in\left[v_{\min }, v_{\max }\right] ; \\
& \chi_{i}(k+s \mid k) \in\left[\chi_{\min }, \chi_{\max }\right] ; \\
& v_{i}^{c}(k+s \mid k) \in\left[v_{\min }, v_{\max }\right] ; \\
& \chi_{i}^{c}(k+s \mid k) \in\left[\chi_{\min }, \chi_{\max }\right] ; \\
& \frac{\left|v_{i}(k+s+1 \mid k)-v_{i}(k+s \mid k)\right|}{\tau} \\
& \in\left[0, \Delta v_{\max }\right] ; \\
& \frac{\left|\chi_{i}(k+s+1 \mid k)-\chi_{i}(k+s \mid k)\right|}{{ }_{i}^{\mathrm{adrl}}\left(x_{i}(k), u_{i}(k)\right)>0 ;} \\
& h_{i}^{\mathrm{ewr}}\left(x_{i}(k), u_{i}(k)\right)>0 ; \\
& \\
& \\
&
\end{aligned}
$$

$$
\begin{aligned}
& h_{i}^{\mathrm{adr} 2}\left(x_{i}(k), u_{i}(k)\right)<0 ; \\
& h_{i}^{i \mathrm{rc1}}\left(x_{i}(k), u_{i}(k)\right)>0 ; \\
& d^{i, j}(k) \geq D_{\text {safe }} \\
& \quad\left(\forall_{i \neq j}, i, j \in\left\{1, \ldots, N_{v}\right\}\right) ; \\
& d^{i, j}(k) \leq D_{\text {com }} \\
& \quad\left(\forall_{i \neq j}, i, j \in\left\{1, \ldots, N_{v}\right\}\right) .
\end{aligned}
$$

\section{Finite State Machine Formation Management Unit}

5.1. Finite State Machine (FSM). Finite state machine is mainly used to describe the conversion process and mechanism of object between different states, and its mathematical definition can be defined as follows: a finite state machine $Q$ can be expressed by a quintet $Q=\{\Sigma, S, f, I, O\} . S=$ $\left\{S_{1}, S_{2}, \ldots, S_{n}\right\}$ is nonempty finite set of state. $I=\left\{I_{1}, I_{2}, \ldots\right.$, $\left.I_{m}\right\}$ is the initial state set, $I \subseteq S$. $O=\left\{O_{1}, O_{2}, \ldots, O_{l}\right\}$ is the final state set, and $O \subseteq S$. $\Sigma$ is the input alphabet (triggering event set). $f$ is mapping function from $S \times \Sigma$ to $S, f: S \times \Sigma \rightarrow S$ [43-46].

5.2. Design of Formation Control Manager Based on FSM. As shown in Figure 6, the adjacent UAVs' state, environmental threat information, task command, and reference trajectory coordinate will be transferred to FSM formation management unit and DMPC formation controller by communication unit. According to this information, FSM formation management unit will make the formation mode of the next step and transfer it to DMPC formation controller, and then the DMPC formation controller will output specific control signal after handling the above information.

The operation steps of the FSM formation management are as follows:

(1) According to the theory of finite state machine and the mission requirement of UAVs formation, we can determine five models of UAV formation as follows: free formation flight $S_{1}$; forming the initial formation $S_{2}$; keeping the formation $S_{3}$; formation reconfiguration $S_{4}$; formation avoidance control $S_{5}$.

(2) According to the formation mode of the first step, the switching condition (trigger event) between two states is determined: UAVs formation command is $I_{1}$; formation satisfies the constraint condition, that is, $I_{2}$; there exists a fixed obstacle or nonfly zone within the predetermined range, that is, $I_{3}$; there exists no fixed obstacle or nonfly zone within the predetermined range, that is, $I_{4}$; a certain UAV joins into the formation, that is, $I_{5}$; a certain UAV leaves the formation, that is, $I_{6}$; start a cooperative task, such as cooperative attack and cooperative interference, that is, $I_{7}$; end a cooperative task, such as cooperative 


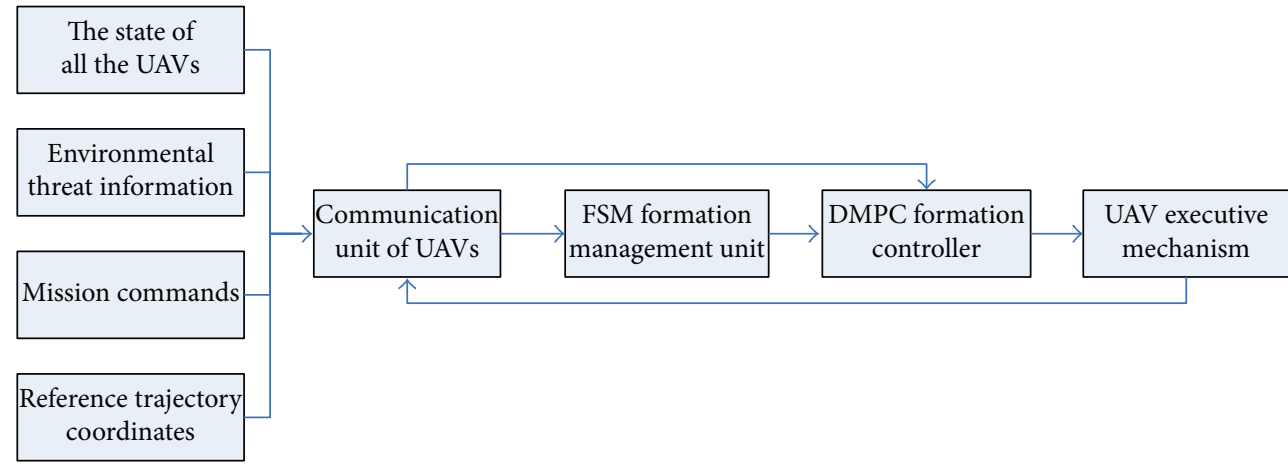

FIGURE 6: Formation control manager based on FSM.

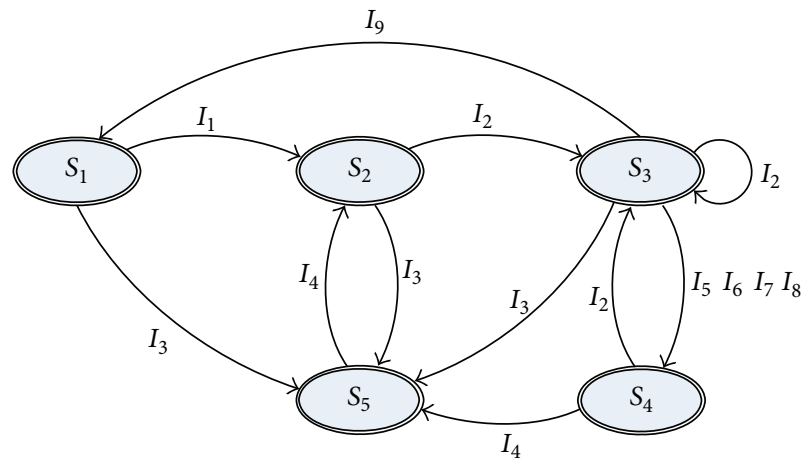

FIGURE 7: FSM state transition diagram.

attack and cooperative interference, that is, $I_{8}$; command of formation dissolution is $I_{9}$.

(3) According to the first step and the second step, we can draw a state transition diagram (Figure 7).

Based on the DMPC formation controller and the FSM formation management unit, this paper presents an automatic formation reconfiguration control method. When UAVs formation arrives in the target zone and finds the early warning radar threat or air defense radar threat, the cooperative interference penetration should be mainly taken. It is usual to select one or several UAVs to interfere in the early warning radar or air defense radar for cooperative interference penetration; for simplicity, in this paper electronic interference will be carried out only by the UAV whose distance is nearest from radar interfere, and the overall formation transforms the formation based on this UAV and reference trajectory point, while it must satisfy the constraints of formation forming which is required by cooperative interference penetration. The other UAVs in the formation which has not interference task will arrive to the latest desired relative position as soon as possible. And when the UAVs formation detects the nonfly zone, cooperative penetration can only be carried out by them, and the overall formation can achieve formation transformation based on reference trajectory and nonfly zone.

Assuming that the initial state of formation is $S_{3}$, when a certain UAV of the formation detects the early warning radar or air defense radar, the formation goes into $S_{4}$, and it will select the most suitable UAV which will carry on realtime resolving to obtain the best position to interfere in the early warning radar or air defense radar; the other UAVs in the formation will also carry on real-time resolving to obtain the desired formation parameters; they will justify whether they are in the range of the enemy early warning radar or air defense radar or not until they are in safety $[39,46]$.

\section{Solution of Reconfiguration Problem}

6.1. Quantum Particle Swarm Optimization Algorithm (QPSO). In the model of quantum particle swarm optimization algorithm, the particle motion state can be described by wave function $\psi(X, t)$ as follows:

$$
|\psi|^{2} d x d y d z=Q d x d y d z
$$

$Q$ is probability density function, and it satisfies the normalization condition:

$$
\int_{-\infty}^{+\infty}|\psi|^{2} d x d y d z=\int_{-\infty}^{+\infty} Q d x d y d z=1 .
$$

The particle motion satisfies the Schrödinger equation in the quantum space:

$$
i \hbar \frac{\partial}{\partial t} \psi(X, t)=\widehat{H} \psi(X, t) .
$$

$\hbar$ is Planck constant; $\widehat{H}$ is Hamiltonian operator which can be described as follows:

$$
\widehat{H}=-\frac{\hbar}{2 m} \nabla^{2}+V(X) .
$$

$m$ is the particle mass, $V(X)$ is particle potential energy, and the model is called $\delta$ potential well. Quantum particle swarm optimization algorithm is mainly based on $\delta$ potential well: assuming that there is a group made up of $M$ particles which are represented as problem solution in $N$ dimensional object search space: namely, $X L(t)=$ $\left\{X L_{1}(t), X L_{2}(t), \ldots, X L_{M}(t)\right\}$. At $t$ time $i$ th particle position is $X L_{i}(t)=\left\{X L_{i, 1}(t), X L_{i, 2}(t), \ldots, X L_{i, N}(t)\right\}, i=1,2, \ldots, M$, it has no velocity vector, the best individual position in the 
group can be expressed as $P_{i}(t)=\left[P_{i, 1}^{l}(t), P_{i, 2}^{l}(t), \ldots, P_{i, N}^{l}(t)\right]$, and the best global position in the group can be expressed as $P^{g}=\left(P_{1}^{g}, P_{2}^{g}, \ldots, P_{N}^{g}\right)$. In order to ensure the convergence of the algorithm, each particle converges to an attractor point $p_{i}=\left(p_{i, 1}, p_{i, 2}, \ldots, p_{i, N}\right)$ which can be expressed as follows:

$$
p_{i . j}(t)=\frac{c_{1} r_{1, j}(t) \cdot P_{i, j}^{l}(t)+c_{2} r_{2, j}(t) \cdot P_{j}^{g}(t)}{c_{1} r_{1, j}(t)+c_{2} r_{2, j}(t)} .
$$

$r_{1, j}, r_{2, j}$ are, respectively, random number between 0 and 1 .

The average best position is introduced in the quantum particle swarm optimization algorithm, which can be defined as the average of the individual best position from all the particles: namely,

$$
\begin{aligned}
& \text { Mbest }=\frac{1}{M} \sum_{i=1}^{M} P_{i}^{l}(t) \\
& \quad=\left(\frac{1}{M} \sum_{i=1}^{M} P_{i, 1}^{l}(t), \frac{1}{M} \sum_{i=1}^{M} P_{i, 2}^{l}(t), \ldots, \frac{1}{M} \sum_{i=1}^{M} P_{i, N}^{l}(t)\right) .
\end{aligned}
$$

$M$ is the group scale.

Then the evolution equation of the quantum particle swarm optimization algorithm can be expressed as

$$
X L_{i}(t+1)=p_{i} \pm b \mid \text { Mbest }-X L_{i}(t) \mid \operatorname{In} \frac{1}{u} .
$$

$u$ is a random number between 0 and $1, b$ is the contractionexpansion coefficient, and the selection of parameter $b$ will affect the performance of the algorithm directly. Generally, when $b$ is reduced from 1 to 0.5 , the result is relatively good, so it can be expressed as follows:

$$
b=1-\frac{t}{T} \times 0.5
$$

$T$ is the maximum iteration number and $t$ is the current iteration number.

Compared with the basic particle swarm optimization algorithm, because the attractor point $p_{i}$ is between $P_{i, j}^{l}$ and $P_{j}^{g}$, the particle will probably appear near $P^{g}$ for the particles which are gathering in $P^{g}$. The quantum particle swarm optimization algorithm has less operator and simplifies the calculation, and to introduce the average best position Mbest, there exists a waiting effect among the particles which can greatly improve the cooperative work capability and enhance the global search capability of the algorithm $[47,48]$.

6.2. Solution of Formation Reconfiguration Based on RQPSOFSM-DMPC. Although QPSO has been proved to be a global convergence algorithm, it may also fall into premature when it is used to deal with complex optimization problem such as multipeak function. In order to improve this problem, Sun et al. introduced the diversity guidance strategy into PSO and proposed the attraction and repulsion particle swarm optimization algorithm [47]. By controlling the diversity of the group, the attraction and repulsion particle swarm optimization algorithm can achieve the switch between the two modes of global search and local search. The attraction corresponded with the convergence phase, and the repulsion corresponded with the divergence phase.

The paper takes the average Euclidean distance between the particle and the centre point to express the diversity. The group made up by the current position of the particle and the group made up by the individual best position of the particle can be expressed as

$$
\begin{gathered}
S_{X}=\left(X_{1}, X_{2}, \ldots, X_{M}\right), \\
S_{P}=\left(P_{1}, P_{2}, \ldots, P_{M}\right) .
\end{gathered}
$$

Thus, the diversity metric can be expressed as follows:

$$
\begin{gathered}
\operatorname{diversity}\left(S_{X}\right)=\frac{1}{M \cdot|A|} \cdot \sum_{i=1}^{M} \sqrt{\sum_{j=1}^{N}\left(X_{i, j}-\bar{X}_{j}\right)^{2}}, \\
\bar{X}_{j}=\frac{1}{M} \sum_{i=1}^{M} X_{i, j}, \\
\operatorname{diversity}\left(S_{P}\right)=\frac{1}{M \cdot|A|} \cdot \sum_{i=1}^{M} \sqrt{\sum_{j=1}^{N}\left(P_{i, j}-\bar{P}_{j}\right)^{2}}, \\
\bar{P}_{j}=\frac{1}{M} \sum_{i=1}^{M} P_{i, j}=C_{j} .
\end{gathered}
$$

$|A|$ is the length of the longest diagonal in the search space, $N$ is the dimension of optimization, and $M$ is the scale of particle swarm:

$$
|A|=\left(X_{\max }-X_{\min }\right) \cdot \sqrt{N} .
$$

Time is critical for the battlefield, so search speed is more important in the search early and middle phase, but when entering the search later phase, the diversity of particle reduces, and the capability of global search will subsequently be greatly degraded. In order to avoid the premature phenomenon effectively, this paper sets the lower limit of the diversity and the QPSO algorithm which is introduced by the diversity is applied to UAVs formation reconfiguration control.

We can use the diversity metrics diversity $\left(S_{X}\right)$ to guide the algorithm search. After the initialization of the particle swarm, it will converge. According to (26), we can know that $b$ will decrease linearly from 1 to 0.5 . In the process of convergence, once diversity $\left(S_{X}\right)$ is smaller than the preset lower limit $d_{\text {low }}$, the particle swarm will enter into the divergence state; this is a temporary increase of diversity until diversity $\left(S_{X}\right)$ is larger than $d_{\text {low }}$.

When $\operatorname{diversity}\left(S_{X}\right)$ or $\operatorname{diversity}\left(S_{P}\right)$ is less than $d_{\text {low }}$, the mutation operation can be carried out for the particles in the best position of the group:

$$
\begin{aligned}
P_{j}^{g}=P_{j}^{g}+\ell \cdot|A| \cdot \varepsilon, & \\
& \varepsilon \sim N(0,1),(j=1,2, \ldots, N) .
\end{aligned}
$$


$\varepsilon$ is the random number according to the standard normal distribution and $\ell$ is the mutation parameter.

By conducting the mutation operation, the distance between the particle individual best position and the average best position can be increased, and then the value of $\operatorname{diversity}\left(S_{P}\right)$ will increase. At the same time, due to the change of the global best position that can make Mbest deviate from the current position, the distance between the particle current position and Mbest will also increase and then will directly lead to a certain degree of divergence of particles which will increase $\operatorname{diversity}\left(S_{X}\right)$. Therefore, we can conduct the mutation by increasing two kinds of diversity $\left(\operatorname{diversity}\left(S_{P}\right)\right.$ or diversity $\left.\left(S_{X}\right)\right)$; the former is called $\mathrm{DGQPSO}_{P}$ and the latter is called DGQPSO ${ }_{X}$.

In addition to carrying out the mutation operation for particle swarm which can increase the diversity of group, this paper proposes another measure to increase the diversity of the group by conducting selection operation on $P^{g}$ which can avoid falling into the local optimal solution. Namely, $p_{i}$ of particles is no longer determined by the global best position and $P_{i}^{l}$ but is determined by $P_{i}^{l}$ and the individual best position of the other particles which are selected randomly. After selecting some other particle $P_{k}^{l}(t)$ from particle swarm we can carry out the following operation:

$$
P^{g}= \begin{cases}P_{k}^{l}(t), & f\left(P_{k}^{l}(t)\right)<f\left(P_{i}^{l}(t)\right), \\ P_{i}^{l}(t), & f\left(P_{k}^{l}(t)\right) \geq f\left(P_{i}^{l}(t)\right) .\end{cases}
$$

When the objective function value which is determined by $P_{k}^{l}(t)$ is better than $P_{i}^{l}(t)$, then $P_{i}(t)$ is determined by $P_{k}^{l}(t)$ and $P_{k}^{l}(t)$; otherwise, it is determined by $P_{g}(t)$ and $P_{i}^{l}(t)$. Therefore, the coordinate of $P_{i}(t)$ can be expressed as follows:

$$
p_{i, j}(t)=\zeta \cdot P_{i, j}(t)+(1-\zeta) \cdot G_{j}(t), \quad \zeta \sim U(0,1) .
$$

The individual optimal position of each particle is likely to be selected as $P^{g}$, so the particle swarm will tend to it. If some nonglobal best position of the particle becomes the target which is the convergence of the particle swarm, and it is located near the global optimal solution, then the probability of finding the global optimal solution will be increased greatly, so that the global search capability of QPSO algorithm is improved evidently $[49,50]$.

The revised quantum particle swarm algorithm (RQPSO) is obtained by introducing the diversity and selection strategy, and its process is described as follows.

Step 1. Set the basic parameters of the algorithm, encode the particles (Figure 8), initialize the particle swarm randomly, and establish the mapping relationship between the problems and particles.

Step 2. Perform the following steps when the iteration number is less than $T$.

Step 3. Calculate the average best position of the particle swarm, namely, Mbest.
Step 4. Calculate the current fitness value of the particles and compare it with the fitness value of the former iteration; if the current fitness value is less than the former iteration, then the particle position is updated by the current particle position; namely, if $f\left(X_{i}(t+1)\right)<f\left(P_{i}(t)\right)$, then $P_{i}(t+1)=X_{i}(t+1)$.

Step 5. Calculate the current global optimal position of the group, namely, $P^{g}$.

Step 6. Compare the fitness value of the current global optimal position with the fitness value of the former global optimal position; if the current global optimal position is better, then the global optimal position of the group is updated by it.

Step 7. Select the point $P^{g}$ for each particle, and calculate $p_{i, j}$ depending on (32) for each dimension of the particle.

Step 8. According to (25), calculate the new position of the particles.

Step 9. Calculate the particle swarm diversity, namely, $\operatorname{diversity}\left(S_{X}\right)$ or diversity $\left(S_{P}\right)$.

Step 10. Calculate the contraction-expansion coefficient and conduct the convergence model.

Step 11. Justify diversity $\left(S_{X}\right)$ or $\operatorname{diversity}\left(S_{P}\right)$; if it is less than $d_{\text {low }}$, then carry out the mutation for the global best particle depending on (30) and conduct the divergence mode.

Step 12. According to the quantum particle swarm optimization evolution equation, update the position for each particle of the group.

Step 13. Return to Step 2.

\section{Simulation and Analysis}

In order to verify the effectiveness of formation reconfiguration control method which is proposed by this paper, we have made procedure simulation under the MATLAB compiler environment, adopt the virtual-leader formation control strategy, and then make UAVs formation reconfiguration control simulation experiment by particle swarm optimization (PSO) algorithm and revise quantum particle swarm optimization (RQPSO) algorithm, respectively. We set the particle swarm scale and the max iteration to be 20 and 400, respectively, in PSO and RQPSO. In PSO, local coefficient and global coefficient are set to 2, and inertia weight is set to 0.5 . In RQPSO, the contraction-expansion coefficient $b$ is reduced linearly from 1 to 0.5 , the lower limit value of diversity is set to 0.0004 , and the parameter $\ell$ is set to 0.000012 .

Assuming the communication link between UAVs is in good condition, the communication delay need not be considered. The velocity and track azimuth of all the UAVs can be measured, and there is no interference which can interfere in the velocity and track azimuth. The parameters of early warning radar, air defense radar, and nonfly zone 


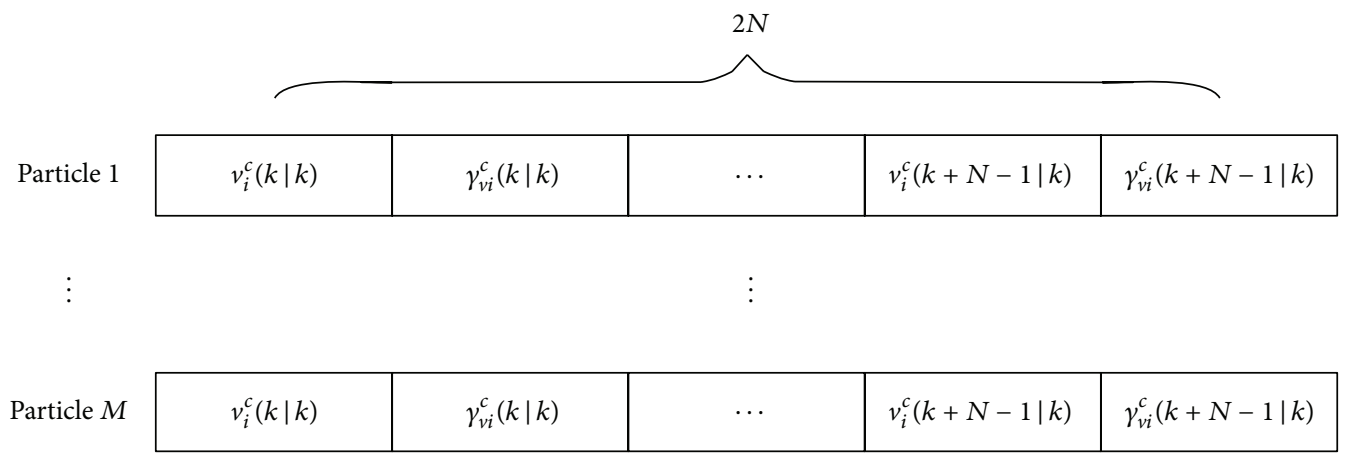

FIGURE 8: Particle encoding.

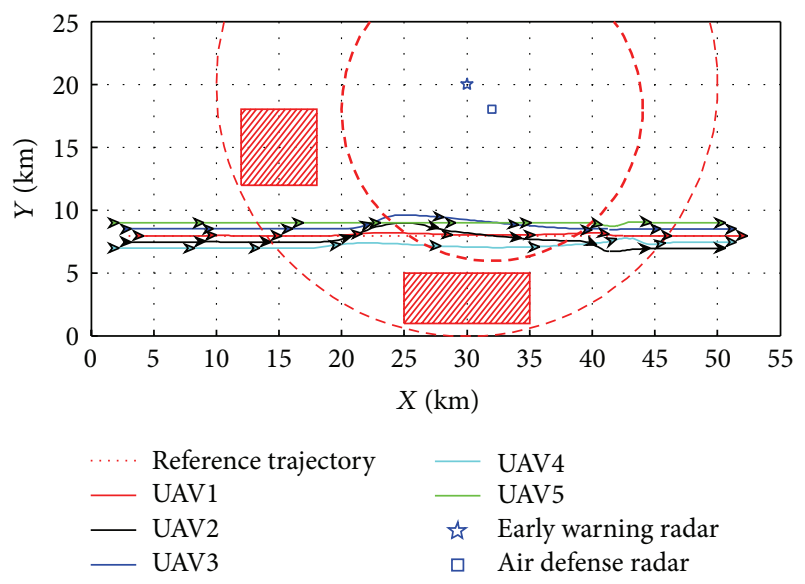

FIGURE 9: Automatic configuration transforming flight trajectory under threaten environment.

and collision avoidance constraints and communication constraints are shown in Table 1; the state transformation is shown in Table 5. The reference trajectory and the UAVs initial motion parameters are shown in Tables 2 and 3 . The relative distance between UAVs in the formation and the reference trajectory is shown in Table 4.

The simulation results are shown in Figures 9-14.

From Figure 9, we can see that the overall UAVs formation does not deviate the reference trajectory too far in the process of flight, and it can automatically switch the formation control law to complete the transformation between formation keeping and formation reconfiguration in accordance with each discrete event. When the UAVs formation detects the enemy early warning radar and carries out penetrating, UAV5 is selected to interfere in it, and the other UAVs regard UAV5 and reference trajectory as reference to transform formation automatically, so that they can be in a safe zone. When the UAVs formation detects the air defense radar tracking threat, UAV3 is selected to interfere in it; at this moment, UAV3 and UAV5 select the optimal interference position cooperatively and automatically, the formation completes the optimum formation solution, and all the UAVs can reach the desired position quickly and realize safe penetration. After successful penetration, according to

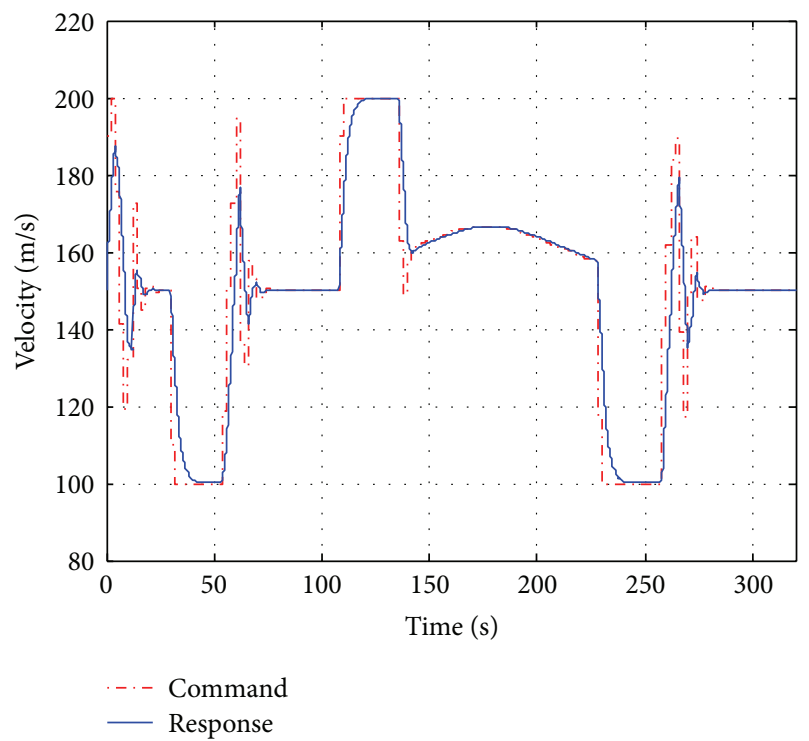

FIGURE 10: Velocity instruction and response of UAV1.

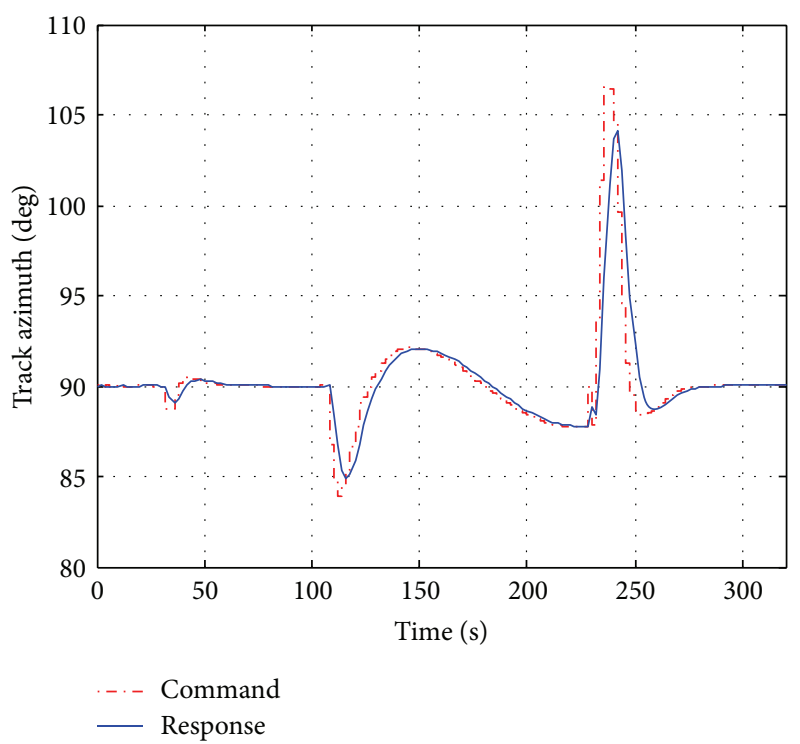

FIGURE 11: Course angle instruction and response of UAV1. 


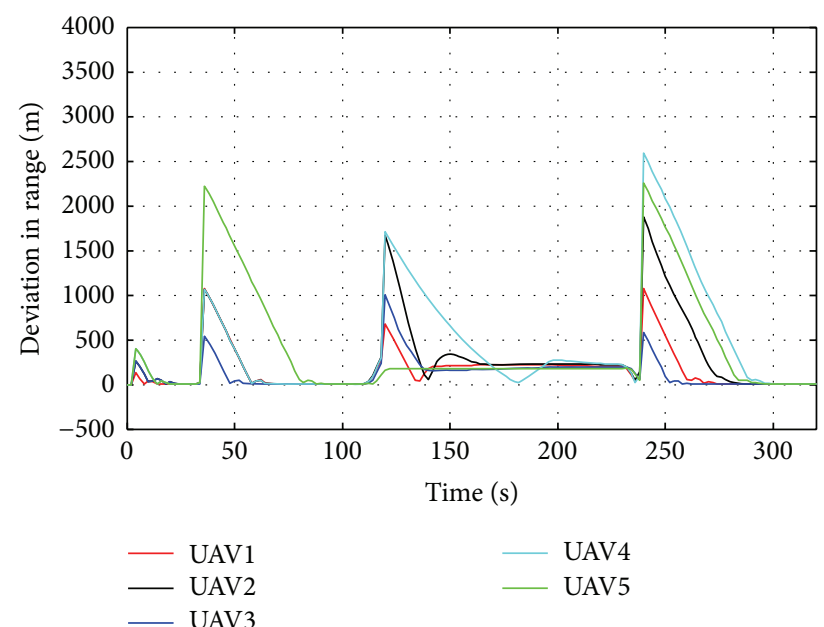

FIGURE 12: Desired location tracking deviation graph of UAVs.

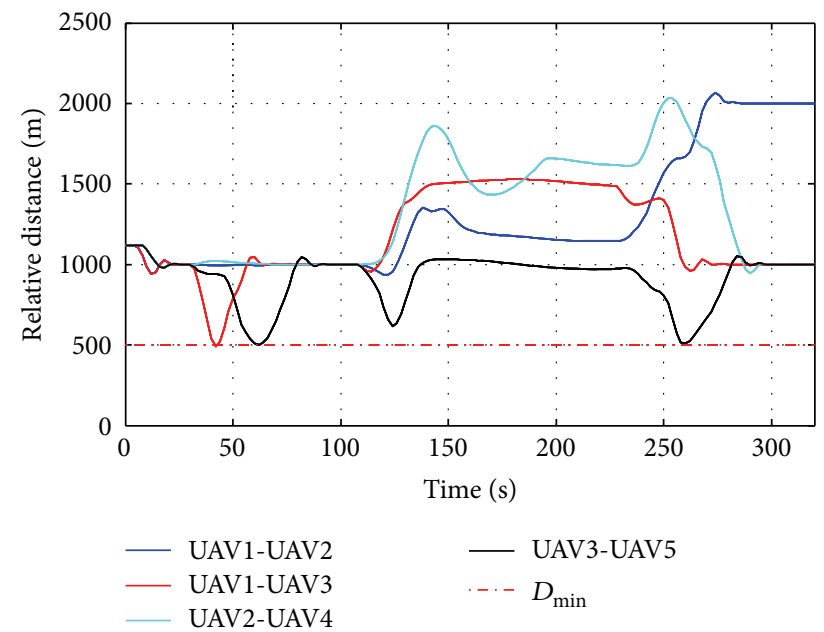

FIgURE 13: Relative distance between UAVs.

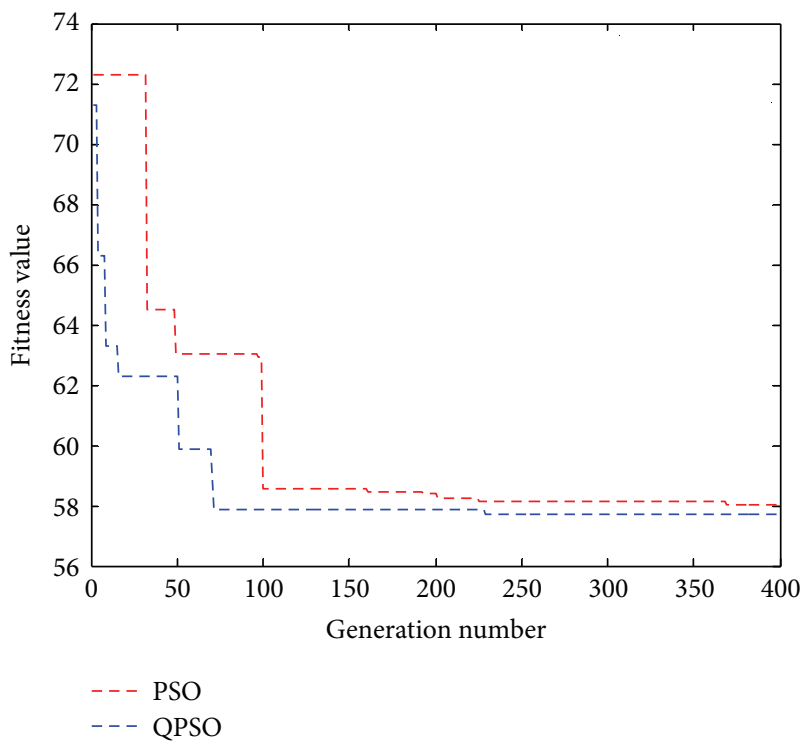

FIGURE 14: The convergence speed between RQPSO and PSO.
TABLE 1: Parameters of enemy threat.

\begin{tabular}{lc}
\hline Parameter & Value \\
\hline $\begin{array}{l}\text { Coordinate and action } \\
\text { distance of early warning }\end{array}$ & $(30,20) 20 \mathrm{~km}$ \\
radar & \\
Coordinate, action & $(32,18) 12 \mathrm{~km} 10^{0}$ \\
distance, and angle of air & \\
defense radar & {$[(12,12),(18,12),(18,18),(12,5)]$} \\
The first nonfly zone & {$[(25,25),(35,25),(35,5),(25,5)]$} \\
The second nonfly zone & $500 \mathrm{~m}$ \\
Distance of collision & $2 \mathrm{~km}$ \\
avoidance & {$[100,200]$} \\
The max communication & {$[80,110]$} \\
distance & {$[2.5,5]$} \\
{$\left[v_{\min }, v_{\max }\right]$} & 20 \\
{$\left[\chi_{\min }, \chi_{\max }\right]$} & 5 \\
{$\left[\omega_{\min }, \omega_{\max }\right]$} & \\
$\Delta v_{\max }$ & \\
$\Delta \chi_{\max }$ &
\end{tabular}

TABLE 2: Parameter of reference trajectory.

\begin{tabular}{lcc}
\hline Parameter & Time & Value \\
\hline Initial position & $0 \mathrm{~s}$ & $(2.8,8.2)$ \\
Velocity & {$[0,200] \mathrm{s}$} & $152 \mathrm{~m} / \mathrm{s}$ \\
Track azimuth & {$[0,200] \mathrm{s}$} & 90 \\
\hline
\end{tabular}

TABLE 3: Initial kinematic parameters of the UAVs.

\begin{tabular}{lccc}
\hline UAVs & $(x, y) / \mathrm{km}$ & $v(\mathrm{~m} / \mathrm{s})$ & $\chi / \mathrm{deg}$ \\
\hline UAV1 & $(3.8,8.2)$ & 152 & 90 \\
UAV2 & $(2.8,7.7)$ & 152 & 90 \\
UAV3 & $(2.8,8.7)$ & 152 & 90 \\
UAV4 & $(1.8,7.2)$ & 152 & 90 \\
UAV5 & $(1.8,9.2)$ & 152 & 90 \\
\hline
\end{tabular}

TABLE 4: Parameters of desired formation configuration.

\begin{tabular}{lc}
\hline Mathematic symbol & Value \\
\hline$\left(x_{1 r}^{d}, y_{1 r}^{d}\right)$ & $(0,1 / \sin (\pi / 3))$ \\
$\left(x_{2 r}^{d}, y_{2 r}^{d}\right)$ & $(0.5,0.5 / \sin (\pi / 6))$ \\
$\left(x_{3 r}^{d}, y_{3 r}^{d}\right)$ & $(-0.5,0.5 / \sin (\pi / 6))$ \\
$\left(x_{4 r}^{d}, y_{4 r}^{d}\right)$ & $(1,-0.5 / \cos (\pi / 6))$ \\
$\left(x_{5 r}^{d}, y_{5 r}^{d}\right)$ & $(-1,-0.5 / \cos (\pi / 6))$ \\
\hline
\end{tabular}

the latest position and the desired formation, the UAVs formation makes the formation reconfiguration; although it forms the same formation with the former, the relative position among the UAVs has changed; this phenomenon shows that the formation which is made up of five UAVs can transform the formation nimbly under the threats environment. Figures 10 and 11 show the velocity, course angle control input of UAV1 and its actual velocity, and course angle response, and from the figures, we can achieve that the actual velocity and course angle response are within the 
TABLE 5: Time step of the state transition.

\begin{tabular}{lcc}
\hline Time & State transition & Trigger event \\
\hline $0 \mathrm{~s}$ & $S_{1} \rightarrow S_{2}$ & $I_{1}$ \\
$12 \mathrm{~s}$ & $S_{2} \rightarrow S_{3}$ & $I_{2}$ \\
$34 \mathrm{~s}$ & $S_{3} \rightarrow S_{4}$ & $I_{8}$ \\
$58 \mathrm{~s}$ & $S_{4} \rightarrow S_{3}$ & $I_{2}$ \\
$66 \mathrm{~s}$ & $S_{3} \rightarrow S_{5}$ & $I_{3}$ \\
$73 \mathrm{~s}$ & $S_{5} \rightarrow S_{2} \rightarrow S_{3}$ & $I_{4}, I_{2}$ \\
$112 \mathrm{~s}$ & $S_{3} \rightarrow S_{4}$ & $I_{8}$ \\
$290 \mathrm{~s}$ & $S_{4} \rightarrow S_{3}$ & $I_{10}, I_{2}$ \\
\hline
\end{tabular}

range of constraint. Figure 12 shows the desired location tracking deviation of five UAVs, and from the figure, we can get that all the tracking deviations are within the range of constraint. Figure 13 shows that the relative distance between one UAV and another meets the constraints; thus, it validates the fact that the formation configuration automatic control method which is designed by this paper is effective. Figure 14 shows that the effect of revised quantum particle swarm optimization algorithm is better than particle swarm algorithm apparently.

\section{Conclusion}

In this paper, a finite state machine management unit scheme was proposed in the framework of distributed model predictive control. Because the formation adopts the virtualleader control model and the battle field created the known threat constraints, such as early warning radar, air defense radar, nonfly zone, collision free constraints, and communication distance constraints, the formation configuration is determined by the reference trajectory point and the above threat constrains. This paper regards the cost of formation reconfiguration as the optimization objective, introduces the finite state machine scheme to justify the future state of the UAVs formation, and then adopts the revised quantum particle swarm optimization algorithm to solve the problem, while comparing it with particle swarm optimization algorithm. Simulation results show that the designed method is able to keep and transform the formation along with the desired reference trajectory when avoiding the threat of battlefield, avoiding intervehicle collision, and meeting the communication distance limit. Furthermore, we will explore the results when there exists communication delay or there exists no communication in a short period of time on the designed formation reconfiguration control problem.

\section{Competing Interests}

The authors declare that they have no competing interests.

\section{References}

[1] Z. Chao, S.-L. Zhou, L. Ming, and W.-G. Zhang, "UAV formation flight based on nonlinear model predictive control,"
Mathematical Problems in Engineering, vol. 2012, Article ID 261367, 15 pages, 2012.

[2] W. Liu, S. Zhou, S. Yan, and G. Yin, "Robust leaderless consensus of uncertain multiagent systems with fast switching topologies," Mathematical Problems in Engineering, vol. 2015, Article ID 810950, 6 pages, 2015.

[3] E. de Vries and K. Subbarao, "Cooperative control of swarms of unmanned aerial vehicles," in Proceedings of the 49th AIAA Aerospace Sciences Meeting Including the New Horizons Forum and Aerospace Exposition, pp. 1-23, AIAA, Orlando, Fla, USA, 2011.

[4] F. Liao and J. L. Wang, "Formation and reconfiguration control for nonholonomic UAVs with velocity constraints," in Proceedings of the AIAA Guidance, Navigation, and Control Conference, AIAA, Minneapolis, Minn, USA, 2012.

[5] S. Zelinski, T. J. Koo, and S. Sastry, "Hybrid system design for formations of autonomous vehicles," in Proceedings of the 42nd IEEE Conference on Decision and Control, pp. 1-6, December 2003.

[6] C. Bai, H. B. Duan, C. Li, and Y. Zhang, "Dynamic multiUAVs formation reconfiguration based on hybrid diversity-PSO and time optimal control," in Proceedings of the IEEE Intelligent Vehicles Symposium, pp. 775-779, Xi'an, China, June 2009.

[7] W. Ren and Y. Chen, "Leaderless formation control for multiple autonomous vehicles," in Proceedings of the Guidance, Navigation, and Control Conference, pp. 505-514, August 2006.

[8] T. Paul, T. R. Krogstad, and J. T. Gravdahl, "UAV formation flight using 3D potential field," in Proceedings of the 16th Mediterranean Conference on Control and Automation, pp. 1240-1245, IEEE, Ajaccio, France, June 2008.

[9] M. Chiaramonti, F. Giulietti, and G. Mengali, "Formation control laws for autonomous flight vehicles," in Proceedings of the 14th Mediterranean Conference on Control and Automation (MED '06), Ancona, Italy, June 2006.

[10] Z. Weihua and T. H. Go, "3-D formulation of formation flight based on model predictive control with collision avoidance scheme," in Proceedings of the 48th AIAA Aerospace Sciences Meeting Including the New Horizons Forum and Aerospace Exposition, Orlando, Fla, USA, January 2010.

[11] J. Z. Sasiadek and D. S. Necsulescu, "Nonlinear control for UAV formation flying," in Proceedings of the 17th World Congress. The International Federation of Automatic Control, vol. 17, Seoul, Republic of Korea, July 2008.

[12] Z. Weihua, T. H. Go, and E. Low, "Formation flight control using model predictive approach," in Proceedings of the 47th AIAA Aerospace Sciences Meeting including the New Horizons Forum and Aerospace Exposition, Orlando, Fla, USA, January 2009.

[13] A. Richards and J. How, "Decentralized model predictive control of cooperating UAVs," in Proceedings of the 43rd IEEE Conference on Decision and Control (CDC '04), vol. 4, pp. 42864291, Atlantis, Bahamas, December 2004.

[14] W. Ren, "On constrained nonlinear tracking control of a small fixed-wing UAV," Journal of Intelligent and Robotic Systems, vol. 48, no. 4, pp. 525-537, 2007.

[15] G. Hattenberger, R. Alami, and S. Lacroix, "Planning and control for unmanned air vehicle formation flight," in Proceedings of the IEEE/RSJ International Conference on Intelligent Robots and Systems (IROS '06), pp. 5931-5936, Beijing, China, October 2006.

[16] G. Hattenberger, S. Lacroix, and R. Alami, "Formation flight: evaluation of autonomous configuration control algorithms," 
in Proceedings of the IEEE/RSJ International Conference on Intelligent Robots and Systems (IROS '07), pp. 2628-2633, IEEE, San Diego, Calif, USA, November 2007.

[17] T. Furukawa, "Time-subminimal trajectory planning for discrete non-linear systems," Engineering Optimization, vol. 34, no. 3, pp. 219-243, 2002.

[18] K. Y. Lee and M. W. M. G. Dissanayake, "Numerical solution for a near-minimum-time trajectory for two coordinated manipulators," Engineering Optimization, vol. 30, no. 3-4, pp. 227-247, 1998.

[19] T. Furukawa, H. F. Durrant-Whyte, F. Bourgault, and G. Dissanayake, "Time-optimal coordinated control of the relative formation of multiple vehicles," in Proceedings of the IEEE International Symposium on Computational Intelligence in Robotics and Automation (CIRA '03), vol. 1, pp. 259-264, July 2003.

[20] T. Siméon, S. Leroy, and J.-P. Laumond, "Path coordination for multiple mobile robots: a resolution-complete algorithm," IEEE Transactions on Robotics and Automation, vol. 18, no. 1, pp. 4249, 2002.

[21] R. O. Saber, W. B. Dunbar, and R. M. Murray, "Cooperative control of multi-vehicle systems using cost graphs and optimization," in Proceedings of the American Control Conference, pp. 2217-2222, IEEE, Denver, Colo, USA, June 2003.

[22] A. Désilles, H. Zidani, and E. Crück, "Collision analysis for an UAV," in Proceedings of the AIAA Guidance, Navigation, and Control Conference, pp. 1-23, August 2012.

[23] T. Paul, T. R. Krogstad, and J. T. Gravdahl, "Modelling of UAV formation flight using 3D potential field," Simulation Modelling Practice and Theory, vol. 16, no. 9, pp. 1453-1462, 2008.

[24] N. Yokoyama, "Decentralized model predictive control for planning three-dimensional conflict-free trajectories," in Proceedings of the AIAA Guidance, Navigation, and Control Conference, pp. 1-20, 2014.

[25] R. Olfati-Saber and R. M. Murray, "Graph rigidity and distributed formation stabilization of multi-vehicle systems," in Proceedings of the 41st IEEE Conference on Decision and Control, pp. 2965-2971, IEEE, Las Vegas, Nev, USA, December 2002.

[26] H. Peng, L. Shen, and H. Zhu, "Multiple UAV cooperative area search based on distributed model predictive control," Acta Aeronautica et Astronautica Sinica, vol. 31, no. 3, pp. 593-601, 2010.

[27] F. A. P. Lie and T. H. Go, "A collision-free formation reconfiguration control approach for unmanned aerial vehicles," International Journal of Control, Automation and Systems, vol. 8, no. 5, pp. 1100-1107, 2010.

[28] A. Hafez and S. Givigi, "Formation reconfiguration of cooperative UAVs via Learning Based Model Predictive Control in an obstacle-loaded environment," in Proceedings of the Annual IEEE Systems Conference (SysCon '16), pp. 1-8, Orlando, Fla, USA, April 2016.

[29] A. Grancharova, E. I. Grøtli, D.-T. Ho, and T. A. Johansen, "UAVs trajectory planning by distributed MPC under radio communication path loss constraints," Journal of Intelligent \& Robotic Systems, vol. 79, no. 1, pp. 115-134, 2014.

[30] X. Zhang, H. Duan, and C. Yang, "Pigeon-inspired optimization approach to multiple UAVs formation reconfiguration controller design," in Proceedings of the IEEE Chinese Guidance, Navigation and Control Conference (CGNCC '14), pp. 2707-2712, Yantai, China, August 2014.

[31] W. Ren, "Trajectory tracking control for a miniature fixed-wing unmanned air vehicle," International Journal of Systems Science, vol. 38, no. 4, pp. 361-368, 2007.
[32] S. Ye, Design and Simulation of the Control Law for UAV in the Air Phase, University of Electronic Science and Technology, Chengdu, China, 2011.

[33] W. Ren, "On constrained nonlinear tracking control of a small fixed-wing UAV," Journal of Intelligent \& Robotic Systems, vol. 48, no. 4, pp. 525-537, 2007.

[34] G. A. S. Pereira, A. K. Das, V. Kumar et al., "Formation control with configuration space constraints," in Proceedings of the IEEE/RJS International Conference on Intelligent Robots and Systems (IROS '03), Las Vegas, Nev, USA, October 2003.

[35] T. Balch and R. C. Arkin, "Behavior-based formation control for multi-robot teams," IEEE Transactions on Robotics and Automation, vol. 14, no. 6, pp. 926-939, 1998.

[36] J. R. T. Lawton, R. W. Beard, and B. J. Young, "A decentralized approach to formation maneuvers," IEEE Transactions on Robotics and Automation, vol. 19, no. 6, pp. 933-941, 2003.

[37] G. Campa, M. R. Napolitano, B. Seanor, and M. G. Perhinschi, "Design of control laws for maneuvered formation flight," in Proceedings of the American Control Conference (AAC '04), pp. 2344-2349, Boston, Mass, USA, July 2004.

[38] J. Shin and H. J. Kim, "Nonlinear model predictive formation flight," IEEE Transactions on Systems, Man, and CyberneticsPart A: Systems and Humans, vol. 39, no. 5, pp. 1116-1125, 2009.

[39] C. Tianli, Z. Chao, Z. Shaolei, and C. Jie, "Automatic formation control of UAVs toward cooperatice penetration," Electronics Optics \& Control, vol. 21, no. 5, pp. 58-62, 2014.

[40] C.-J. Ru, R.-X. Wei, J. Dai, D. Shen, and L.-P. Zhang, "Autonomous reconfiguration control method for UAV's formation based on Nash bargain," Acta Automatica Sinica, vol. 39, no. 8, pp. 1349-1360, 2013.

[41] L. Giovanini and J. Balderud, "Game approach to distributed model predictive control," in Proceedings of the International Control Conference, IET Control Theory \& Applications, pp. 1729-1739, Institution of Engineering and Technology, 2006.

[42] E. Camponogara, D. Jia, B. H. Krogh, and S. Talukdar, "Distributed model predictive control," IEEE Control Systems Magazine, vol. 22, no. 1, pp. 44-52, 2002.

[43] A. Karimoddini, G. Cai, B. M. Chen, H. Lin, and T. H. Lee, "Hierarchical control design of a UAV helicopter," in Advances in Flight Control Systems, InTech, Vienna, Austria, 2011.

[44] A. Karimoddini, H. Lin, B. M. Chen, and T. H. Lee, "Hybrid formation control of the unmanned aerial vehicles," Mechatronics, vol. 21, no. 5, pp. 886-898, 2011.

[45] A. Karimoddini, H. Lin, B. M. Chen, and T. H. Lee, "Hybrid 3-d formation control for unmanned helicopters," Tech. Rep. NUSACT-11-005-Ver.1, Advanced Control Technology Laboratory, 2011.

[46] A. Karimoddini, H. Lin, B. M. Chen, and T. H. Lee, "Hybrid three-dimensional formation control for unmanned helicopters," Automatica, vol. 49, no. 2, pp. 424-433, 2013.

[47] J. Sun, W. Xu, and J. Liu, "Parameter selection of quantumbehaved particle swarm optimization," in Advances in Natural Computation, L. Wang, K. Chen, and Y. S. Ong, Eds., vol. 3612 of Lecture Notes in Computer Science, pp. 543-552, Springer, New York, NY, USA, 2005.

[48] F. Wei, S. Jun, X. Zhenping, and X. Wenbo, "Convergence analysis of quantum-behaved particle swarm optimization algorithm and study on its control parameter," Acta Physica Sinica, vol. 59, no. 6, pp. 3688-3696, 2010.

[49] N. Dong, Z.-Q. Chen, Q.-L. Sun, and Z.-Z. Yuan, "Particleswarm optimization algorithm for model predictive control 
with constraints," Control Theory and Applications, vol. 26, no. 9, pp. 965-969, 2009.

[50] L. Zhang, D.-B. Wang, Y.-H. Gao, and H.-B. Duan, "Study on uninhabited combat air vehicle formation tasks scheduling method based on particle swarm optimization algorithm," Systems Engineering and Electronics, vol. 31, no. 2, pp. 439-442, 2009. 


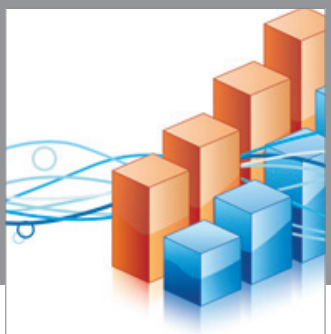

Advances in

Operations Research

vatem alat4

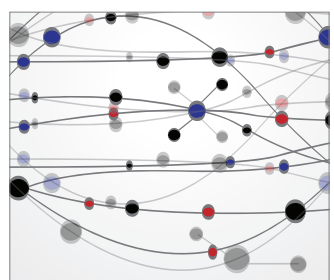

\section{The Scientific} World Journal
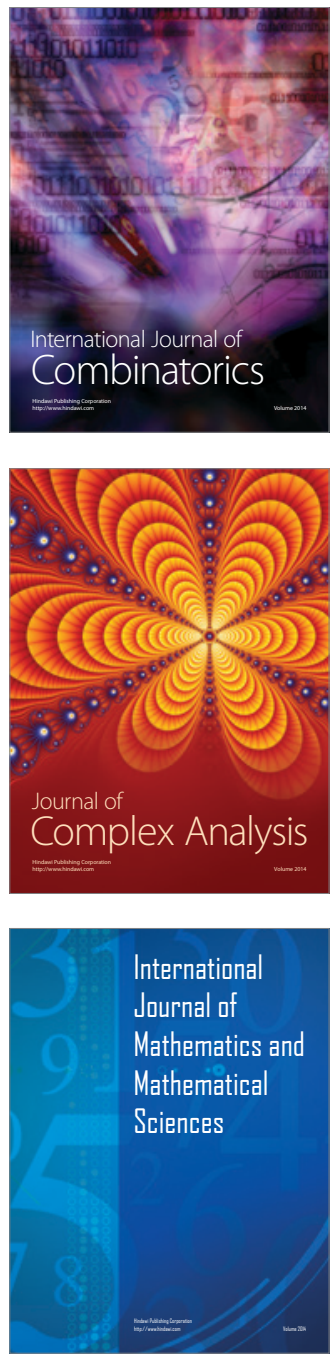
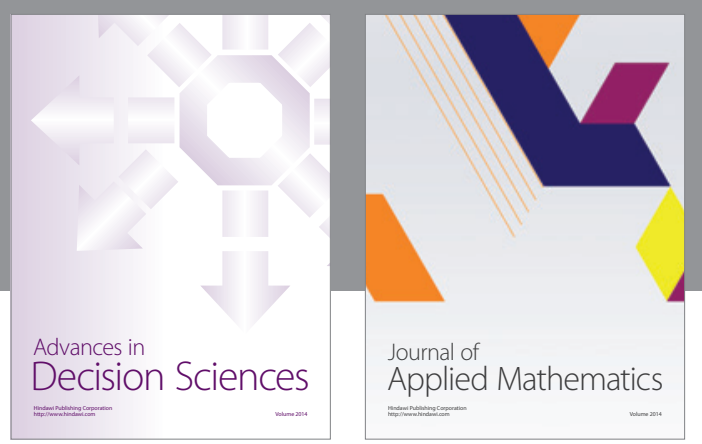

Algebra

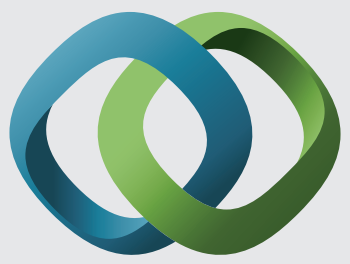

\section{Hindawi}

Submit your manuscripts at

http://www.hindawi.com
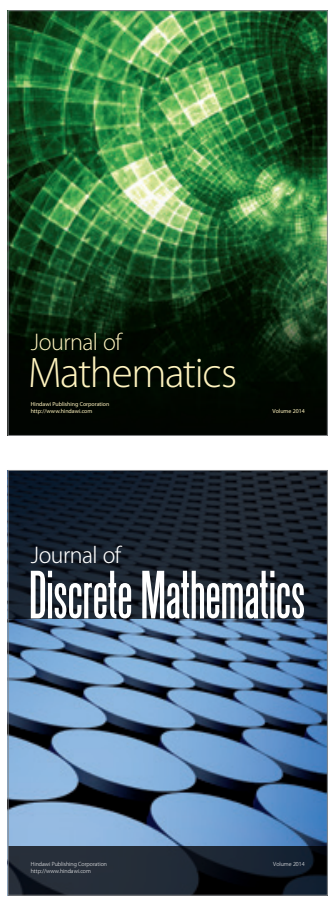

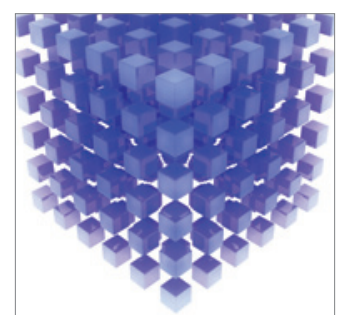

Mathematical Problems in Engineering
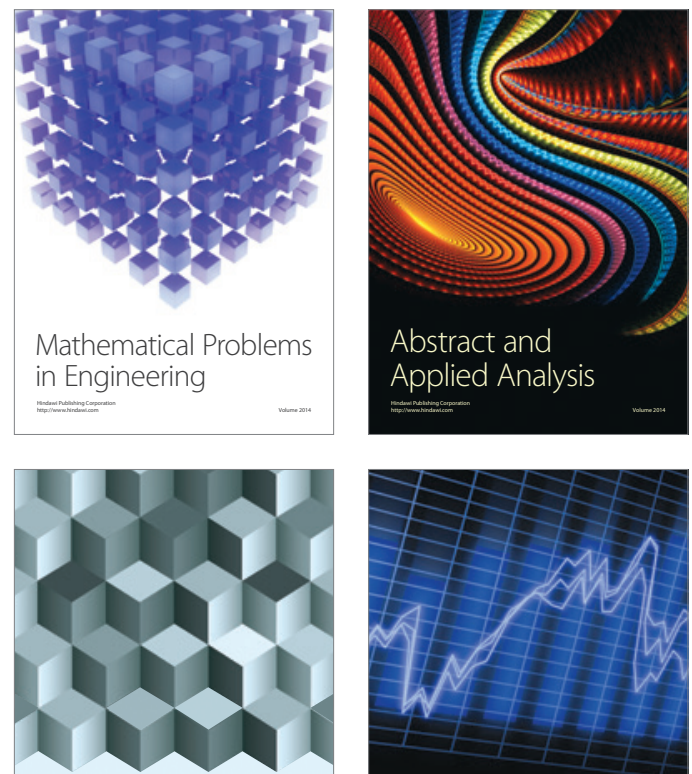

Journal of

Function Spaces

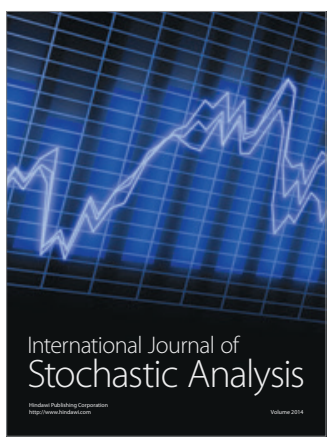

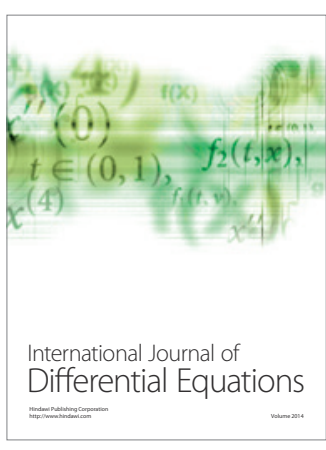
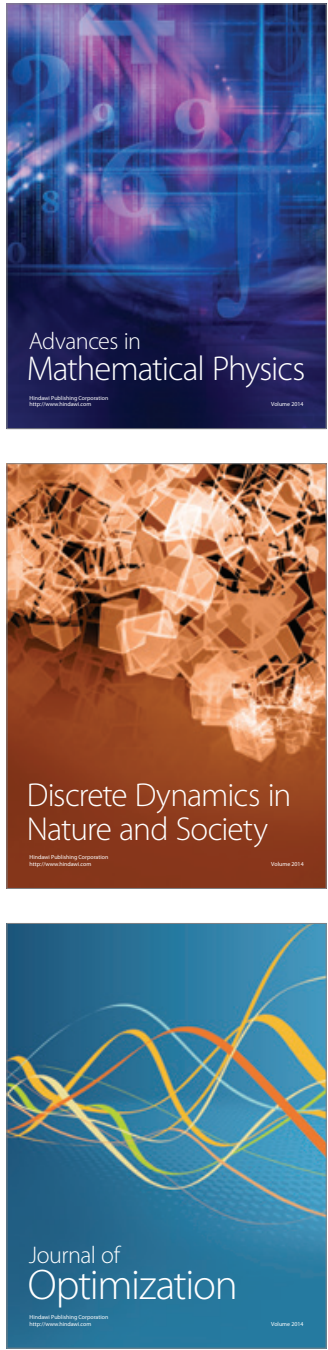